\title{
Revealing the velocity structure of the filamentary nebula in NGC 1275 in its entirety
}

\author{
M. Gendron-Marsolais, ${ }^{1 \star}$ J. Hlavacek-Larrondo, ${ }^{1}$ T. B. Martin, ${ }^{2}$ L. Drissen,,${ }^{2,3}$ \\ M. McDonald, ${ }^{4}$ A. C. Fabian, ${ }^{5}$ A. C. Edge, ${ }^{6}$ S. L. Hamer, ${ }^{7}$ B. McNamara ${ }^{8,9}$ and \\ G. Morrison ${ }^{10}$ \\ ${ }^{1}$ Département de Physique, Université de Montréal, Montréal (Québec), QC H3C 3J7, Canada \\ ${ }^{2}$ Département de physique, de génie physique et d'optique, Université Laval, 1045 avenue de la médecine, Québec (Québec), G1V 0A6, Canada \\ ${ }^{3}$ Department of Physics and Astronomy, University of Hawaii at Hilo, 200 W Kawili Street, Hilo, HI 96720, USA \\ ${ }^{4}$ Kavli Institute for Astrophysics and Space Research, MIT, Cambridge, MA 02139, USA \\ ${ }^{5}$ Institute of Astronomy, University of Cambridge, Madingley Road, Cambridge CB3 OHA, UK \\ ${ }^{6}$ Centre for Extragalactic Astronomy, Department of Physics, Durham University, Durham DH1 3LE, UK \\ ${ }^{7}$ CRAL, Observatoire de Lyon, CNRS, Université Lyon 1, 9 Avenue Charles André, F-69561 Saint Genis-Laval, France \\ ${ }^{8}$ Department of Physics and Astronomy, University of Waterloo, Waterloo, ON N2L 3G1, Canada \\ ${ }^{9}$ Perimeter Institute for Theoretical Physics, Waterloo, ON N2L 2Y5, Canada \\ ${ }^{10}$ LBT Observatory, University of Arizona, 933 N. Cherry Avenue, Room 552, Tucson, AZ 85721, USA
}

Accepted 2018 May 4. Received 2018 May 4; in original form 2018 January 30

\begin{abstract}
We have produced for the first time a detailed velocity map of the giant filamentary nebula surrounding NGC 1275, the Perseus cluster's brightest galaxy, and revealed a previously unknown rich velocity structure across the entire nebula. These new observations were obtained with the optical imaging Fourier transform spectrometer SITELLE at CFHT. With its wide field of view ( $\sim 11 \mathrm{arcmin} \times 11 \mathrm{arcmin})$, SITELLE is the only integral field unit spectroscopy instrument able to cover the $80 \mathrm{kpc} \times 55 \mathrm{kpc}$ ( 3.8 arcmin $\times 2.6$ arcmin) large nebula in NGC 1275. Our analysis of these observations shows a smooth radial gradient of the [NII] $65583 / \mathrm{H} \alpha$ line ratio, suggesting a change in the ionization mechanism and source across the nebula. The velocity map shows no visible general trend or rotation, indicating that filaments are not falling uniformly onto the galaxy, nor being uniformly pulled out from it. Comparison between the physical properties of the filaments and Hitomi measurements of the X-ray gas dynamics in Perseus is also explored.
\end{abstract}

Key words: Galaxies: Individual: NGC 1275 -Galaxies: clusters: individual: Perseus cluster.

\section{INTRODUCTION}

The central dominant galaxy of the Perseus galaxy cluster, NGC 1275 , is surrounded by a giant filamentary emission-line nebula. Such nebulae, with $\mathrm{H} \alpha$ luminosities as high as several $10^{42} \mathrm{erg} \mathrm{s}^{-1}$, are not rare among clusters having peaked X-ray surface brightness distributions like Perseus, known as cool core clusters (e.g. Crawford et al. 1999). However, the filamentary nebula in NGC 1275 extends over $80 \mathrm{kpc} \times 55 \mathrm{kpc}(3.8 \operatorname{arcmin} \times 2.6 \operatorname{arcmin})$ and is therefore among the largest known in any cluster (e.g. McDonald, Veilleux \& Rupke 2012; Hamer et al. 2016). The origin of these nebulae (residual cooling flow or dragged gas) and source of ionization (heat conduction from the intracluster medium [ICM], shocks, or turbulent mixing, e.g. Lim et al. 2012; Fabian 2012) are not yet

^E-mail: marie-lou@astro.umontreal.ca clear. The ionization source does not appear to be related to star formation as the line ratios are different from those in $\mathrm{H}$ II regions (e.g. Kent \& Sargent 1979). These nebulae therefore constitute an active area of research for our understanding of how phenomena such as shocks heat and ionize their surrounding medium.

Being the cluster's brightest galaxy (BCG) in Perseus, NGC 1275 resides in a complex environment, both internally perturbed by the nuclear outbursts of its active galactic nuclei (AGN) and externally affected by interactions with its surrounding environment. As the brightest cluster in the X-ray sky (Forman et al. 1972), it has been observed across all the electromagnetic spectrum, revealing a variety of structures. X-ray observations of the ICM have shown a succession of cavities created by the jets of the central supermassive black hole, pushing away the cluster gas and leaving buoyantly rising bubbles filled with radio emission (e.g. Fabian et al. 2011).

First observed by Minkowski (1957) and Lynds (1970), the optical line-emitting gas towards NGC 1275 consists of two distinct 
components: a high-velocity system $\left(\sim 8200 \mathrm{~km} \mathrm{~s}^{-1}\right)$ corresponding to an infalling foreground galaxy, and a low-velocity system $\left(\sim 5200 \mathrm{~km} \mathrm{~s}^{-1}\right)$ corresponding to the nebula associated with NGC 1275. HST observations reveal a thread-like filamentary composition, some only 70-pc wide and 6-kpc long (Fabian et al. 2008). The brighter filaments have soft X-ray counterparts (Fabian et al. 2003), and Karl G. Jansky Very Large Array 230-470 MHz observations show a spur of emission in the direction of the northern filament (Gendron-Marsolais et al. 2017). Cooler molecular gas is associated with nearly all the filaments, e.g. CO (Salomé et al. 2006, 2011; Ho, Lim \& Dinh-V-Trung 2009) and $\mathrm{H}_{2}$ (Lim et al. 2012).

The nebula was imaged by Conselice, Gallagher \& Wyse (2001) in its full extent with high-resolution imaging, integral field, and long-slit spectroscopy (WIYN \& KPNO). The authors produced a first velocity map of the central $\sim 45 \operatorname{arcsec}(16 \mathrm{kpc}$ ), revealing evidence for rotation, and suggested that the filaments were being formed through compression of the ICM by the AGN outflows. Gemini Multi-Object Spectrograph observations along six slits aligned with 2-3 filaments showed evidence of outflowing gas and flow patterns (Hatch et al. 2006). Overall, these observations suggest that these nebulae could be formed by gas being dragged out from the rise of AGN radio bubbles and stabilized by magnetic fields (Fabian et al. 2003, 2008; Hatch et al. 2006). This argument is further supported by the presence of a horseshoe-shaped filament, bending behind the North-West outer cavity, similar to the toroidal flow pattern trailing behind a buoyant gas bubble in a liquid. Under this assumption, the loop-like X-ray structure extending at the end of the northern filament would then be a fallback of gas dragged out to the north by previously formed bubbles (Fabian et al. 2011). However, the $\mathrm{H} \alpha$ emission found in several cool core clusters' BCGs is delimited within their cooling radius and a strong correlation has been found between $\mathrm{H} \alpha$ luminosity and the X-ray cooling flow rate of the host cluster (McDonald et al. 2010). This suggests that the ionized gas may be linked to the ICM and a radially infalling cooling flow model is favoured.

NGC 1275 is one of the richest nebulae to study due to its proximity and the complexity of its structures. In this article, we present new observations of NGC 1275 obtained with SITELLE, a new optical imaging Fourier transform spectrometer at Canada-FranceHawaii Telescope (CFHT). Unlike previous IFU observations of NGC 1275, its wide field of view (11 arcmin $\times 11$ arcmin ) covers the large nebula in its entirety. To directly compare our results with Hitomi Collaboration et al. (2017), we adopt a redshift of $z$ $=0.017284$ for NGC 1275 , corresponding to an angular scale of $21.2 \mathrm{kpc} \operatorname{arcmin}^{-1}$. This corresponds to a luminosity distance of $75.5 \mathrm{Mpc}$, assuming $H_{0}=69.6 \mathrm{kms}^{-1} \mathrm{Mpc}^{-1}, \Omega_{\mathrm{M}}=0.286$ and $\Omega_{\mathrm{vac}}=0.714$.

\section{DATA REDUCTION AND ANALYSIS}

NGC 1275 was observed in 2016 January with the optical imaging Fourier transform spectrometer SITELLE at CFHT during Queued Service Observations 16BQ12 in science verification mode (PI G. Morrison) with the SN3 filter ( $>90$ per cent transmission from 647 to $685 \mathrm{~nm}$ ) for $2.14 \mathrm{~h}$ (308 exposures of $25 \mathrm{~s}, R=1800)$. SITELLE is a Michelson interferometer with a large field of view (11 arcmin $\times 11$ arcmin, compared to 1 arcmin $\times 1$ arcmin for MUSE and up to 8 arcsec $\times 8$ arcsec for SINFONI) equipped with two E2V detectors of $2048 \times 2064$ pixels, resulting in a spatial resolution of $0.321 \operatorname{arcsec} \times 0.321$ arcsec. These observations were centred at RA $03 \mathrm{~h} 19 \mathrm{~m} 53.19 \mathrm{~s}$ and Dec. $+41^{\circ} 33^{\prime} 51.0^{\prime \prime}$, offset by

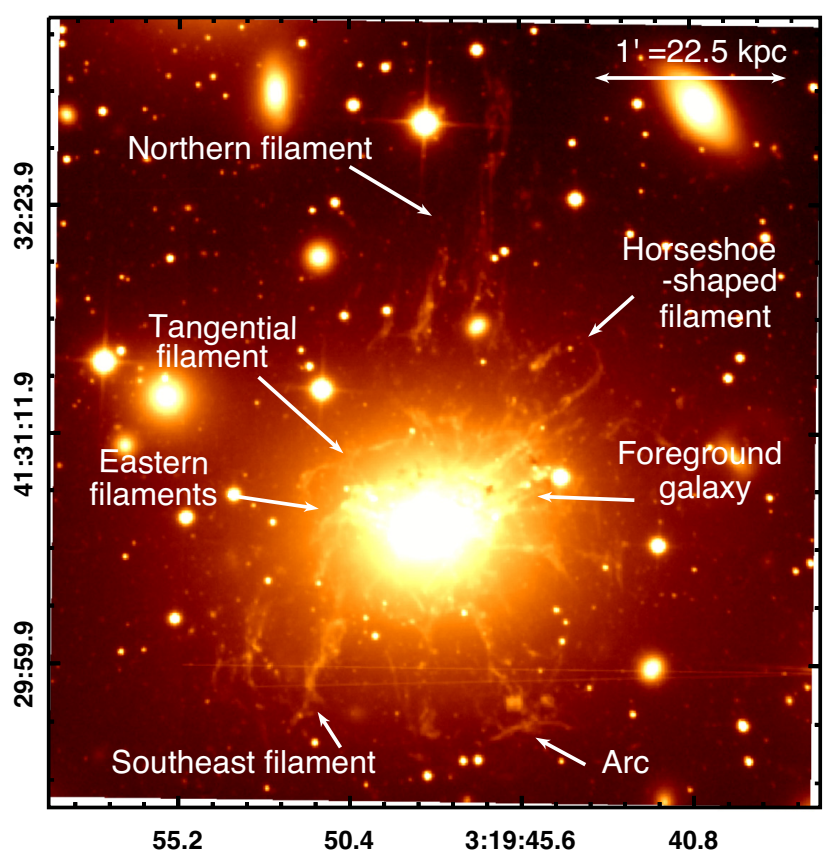

Figure 1. Mean integrated flux SN3 filter image centred on NGC 1275.

about 3 arcmin from NGC 1275 . The data reduction and calibration of these observations were conducted using the SITELLE's software ORCS (version 3.1.2, Martin, Drissen \& Joncas $(2015)^{1}$ ). Five emission lines are resolved in these observations: [N II] $6548, \mathrm{H} \alpha$, $\left[\mathrm{NII}_{\mathrm{II}}\right] \lambda 6584,\left[\mathrm{~S}_{\mathrm{II}}\right] \lambda 6716$, and $\left[\mathrm{S}_{\mathrm{II}}\right] \lambda 6731$. Details of the wavelength and astrometric and photometric calibration followed are described in Martin, Drissen \& Melchior (2018). The OH sky lines velocities were fitted with an optical model of the interferometer in most regions of the cube with the function and the resulting wavelength corrections for instrumental flexures were applied to the cube using SPECTRALCUBE.CORRECT_WAVELENGTH() (Martin et al. 2018). The mean integrated flux SN3 filter image centred on NGC 1275 is shown in Fig.1.

The complexity of this nebula arises from its several components: overlapping filaments with slightly different velocity shifts, the foreground galaxy and the AGN contribution. As we focus only on the nebula component, pixels with $\left[\mathrm{N}_{\mathrm{II}}\right] \lambda 6548, \mathrm{H} \alpha$, and $[\mathrm{NII}] \lambda 6584$ emission lines with a velocity shift close to the NGC 1275 systemic velocity were identified. The foreground galaxy was identified similarly, using an $8200 \mathrm{~km} \mathrm{~s}^{-1}$ systemic velocity, and subtracted. Fitting both the contribution from the AGN and the filaments, we found that the contribution from the AGN is predominant in terms of lines fluxes inside a radius of 6 arcsec. The central region centred at $3 \mathrm{~h} 19 \mathrm{~m} 48.1 \mathrm{~s}+41 \mathrm{~d} 30 \mathrm{~m} 42 \mathrm{~s}$ with a radius of 6 arcsec was excluded. To increase the signal-to-noise without losing too much spatial resolution, we chose to bin the cube by a factor of 2 . The spectrum extracted from each binned remaining pixel was fitted using a Gaussian function convolved with the instrumental line shape - a sinc function (Martin, Prunet \& Drissen 2016). The fitting software uses a least-squares Levenberg-Marquardt minimization algorithm (Levenberg 1944; Marquardt 1963) to fit the data. We restricted the range of wavelengths to the band where $[\mathrm{N}$ II $] \lambda 6548$, $\mathrm{H} \alpha$, and $[\mathrm{NII}] \lambda 6584$ lines are found with the systemic velocity

${ }^{1}$ https://github.com/thomasorb/orcs 
shift of NGC 1275. Sky subtraction was done using the mean flux from a circular region with a radius of 20 arcsec centred at RA 03h19m58.57s and Dec. $+41^{\circ} 30^{\prime} 08.9^{\prime \prime}$, about 2 arcmin south-east of NGC 1275 nucleus. The lines were fitted simultaneously and the velocity and broadening of the three lines grouped to reduce the number of parameters to fit. Only pixels with fitted $\mathrm{H} \alpha$ flux higher than $30 \times 10^{-18} \mathrm{erg} / \mathrm{s} / \mathrm{cm}^{2} /$ pixel were selected. Typical resulting uncertainties on the velocities vary from $\sim 5$ to $\sim 20 \mathrm{~km} \mathrm{~s}^{-1}$. To directly compare our results with Hitomi Collaboration et al. (2017), bulk velocities are calculated with respect to their redshift measurement: $v_{\text {bulk }} \equiv(z-0.017284) * c_{0}-21.9 \mathrm{kms}^{-1}$, where $c_{0}$ is the speed of light and $-21.9 \mathrm{~km} \mathrm{~s}^{-1}$ is the heliocentric correction based on the average value over the observation period from Astropy SKYCOORD.RADIAL_VELOCITY_CORRECTION().

\section{RESULTS AND DISCUSSION}

\subsection{Ionization mechanism}

Fig. 2 (left) shows the $\mathrm{H} \alpha$ flux map. While the surface brightness of $\mathrm{H} \alpha$ is mostly constant in the extended filaments, it is higher in the inner $\sim 30$ arcsec $=11 \mathrm{kpc}$.

Optical line ratios can be good indicators of the dominant excitation mechanism operating on the line-emitting gas (photoionization by stars, by a power-law continuum source or shock-wave heating, Baldwin, Phillips \& Terlevich 1981). The ratio [N II] $66583 / \mathrm{H} \alpha$ provides, e.g a measure of the ionization state of a gas. When the source of ionization is stellar formation, this line ratio is a linear function of metallicity saturating at a value of $\sim 0.5$ for high metallicity (e.g. Kewley et al. 2006). The SITELLE [N II] $\lambda 6583 / \mathrm{H} \alpha$ line ratio map of NGC 1275 is presented in Fig.2 (middle) and the mean and ensemble ratio profiles taken in annuli containing 400 pixels are shown in Fig. 3. The line ratio varies through the map, being $\sim 0.5$ to -1 in the extended filaments, and above 1 in the central part of the nebula. However, streaks of star-forming clusters associated with some filaments of NGC 1275 have been found (Canning et al. 2014). Similar line ratio gradients have also been previously observed in the filaments of NGC 1275 (Hatch et al. 2006) and in several BCG with optical line emission (e.g. Hamer et al. 2016). The central region with higher line ratios could be related to energetic sources of ionization such as AGN and shocks, while filaments must be ionized by a source with lower power. To effectively distinguish the source of ionization though, other line ratios are required, falling outside of the filter used during these observations. The complete detailed Baldwin-Phillips-Terlevich (BPT) diagnostic of NGC 1275 nebula will be conducted using awarded SITELLE observations at 365 385nm and 480-520nm (PI: Gendron-Marsolais) and presented in future work (Gendron-Marsolais et al. in preparation).

\subsection{Velocity dispersion measure across the nebula}

According to the top-down multiphase condensation model, warm filaments and cold molecular clouds condensed out of the hot ICM through 'chaotic cold accretion' (e.g. Gaspari, Temi \& Brighenti 2017b). This link between ICM and filaments implies that both must have the same ensemble velocity dispersion. On the other hand, if these filaments are dragged out from the rise of AGN radio bubbles, they would be stabilized into the hot gas by magnetic fields and therefore also sharing the same velocity field (Fabian et al. 2008). The Hitomi Soft X-ray Spectrometer has shown that the velocity dispersions are on the order of $164 \pm 10 \mathrm{~km} \mathrm{~s}^{-1}$ in the $30-60 \mathrm{kpc}$ region around the nucleus of the Perseus cluster (Hitomi collaboration 2016), while SITELLE has provided an ensemble velocity dispersion of $137 \pm 20 \mathrm{~km} \mathrm{~s}^{-1}$ (Gaspari et al. 2017a). In contrast, the dispersion map obtained from the fitting of each binned pixel shown in Fig. 2 shows instead smaller velocity dispersion in the filaments ( $\lesssim 115 \mathrm{~km} \mathrm{~s}^{-1}$ ) but increasing linewidth closer to the centre (up to $\sim 130 \mathrm{~km} \mathrm{~s}^{-1}$ ). SITELLE's level of resolution therefore probes smaller structures like individual filaments, rather than the ensemble multiphase gas. The mean and ensemble dispersion profiles in Fig. 3 show a general decrease up to $\sim 10 \mathrm{kpc}$ from the nucleus. We note, however, that the inner region contains many overlapping filaments, increasing the measured velocity dispersion. A bump is visible in the profile between $\sim 15$ and $20 \mathrm{kpc}$, corresponding to the region between the inner and ghost cavities and containing a known shock in the ICM to the north-east (Fabian et al. 2006), which could be responsible for the higher mean dispersion.

Further comparisons can be explored between Hitomi and Sitelle velocity dispersions in the same regions as the ones used in Hitomi Collaboration et al. (2017) (see Fig. 4). SITELLE pixels contained in each regions with $\mathrm{H} \alpha$ and [NII] lines (including the AGN but excluding the HV system) were fitted as ensembles (see Table 1). The resulting velocities dispersions are as low $\left(\sim 120 \mathrm{~km} \mathrm{~s}^{-1}\right)$ and uniform as the Hitomi measurements for the hot gas.

\subsection{Kinematics of the filaments}

The velocity map of the nebula (see Fig. 5) reveals a previously unknown rich velocity structure across the entire nebula. The presence of a larger scale velocity gradient is hard to extract from such a detailed map. We note that the median heliocentric velocity of the map is $5229 \mathrm{~km} \mathrm{~s}^{-1}$, giving a high fraction of redshifted pixels ( $\sim 80$ percent) relative to our chosen rest frame. We will discuss this difference in future work (Gendron-Marsolais et al. in preparation). Overall, the mean and ensemble velocity profiles from Fig.3 do not show any clear radial gradient in velocity. On average, the filaments do not seem to be falling smoothly and uniformly onto the galaxy, nor do they seem to be pulled out of it. No potential rotation, as suggested in Conselice et al. (2001), is visible. The lack of ordered motion and the low measured velocities might indicate that these features are short lived, with lifetimes on order of the dynamical time-scale, consistent with the molecular gas (e.g. Lim, Ao \& Dinh-V-Trung 2008, Russell et al. 2016, in the case of the central galaxy of the cluster PKS 0745-191).

With the spectral and spatial resolution provided by SITELLE, the kinematics of the filaments can be studied individually. Velocities from each pixel across the northern filament are plotted against their distance from the AGN in Fig. 6 and show a scattered profile. The mean velocity profile taken in 10 bins of equal width shows a more general trend, varying from positive to negative velocity with the radial distance to the nuclei increase. This is consistent with Gemini Multi-Object Spectrograph observations along this filament (Hatch et al. 2006). The northern filament is therefore either stretching or collapsing depending on its de-projected orientation. The southeast filament also shows complex dynamics with a mostly negative velocity, varying from $\sim-100 \mathrm{~km} \mathrm{~s}^{-1}$ to $-300 \mathrm{~km} \mathrm{~s}^{-1}$ and increasing again up to $\sim 0 \mathrm{~km} \mathrm{~s}^{-1}$ as the distance from the centre increases (see Fig.6). The mean velocity profile across the horseshoe-shaped filament extracted from the bins is shown in Fig.6. It has an overall positive velocity increasing almost symmetrically on either side of the loop, reaching velocities of $\sim 200 \mathrm{~km} \mathrm{~s}^{-1}$. This is similar to the results in Hatch et al. (2006) and consistent with simulations of flow patterns below a rising bubble where the 

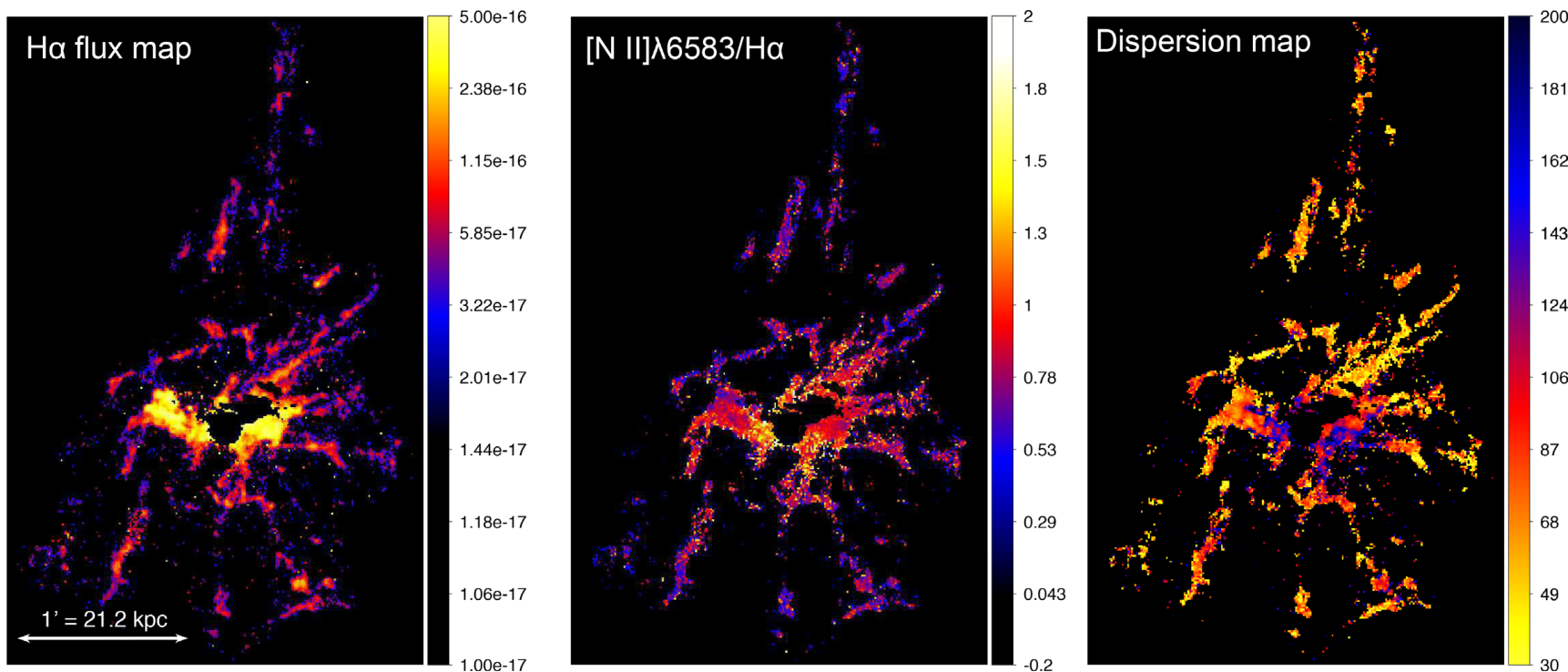

Figure 2. Flux map of $\mathrm{H} \alpha$ emission in the nebula (left, units are in erg/s/ $\mathrm{cm}^{2} /$ pixel), [N II] $\lambda 6583 / \mathrm{H} \alpha$ line ratio map (middle) and dispersion map (right, scale unit is in $\mathrm{km} \mathrm{s}^{-1}$ ).
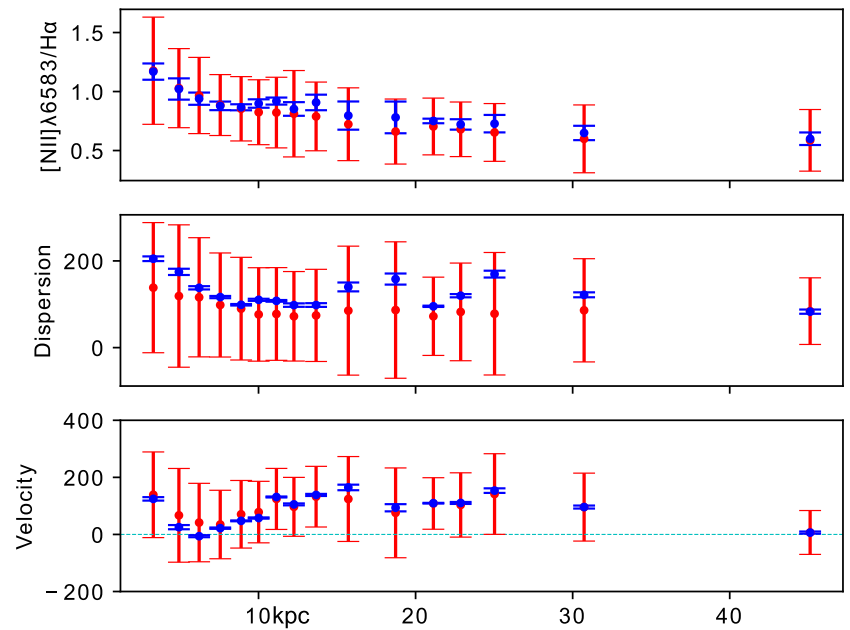

Figure 3. The mean (in red, with error bars indicating the standard deviation) and ensemble fit result (in blue) $[\mathrm{N}$ II] $]$ 6583/ $\mathrm{H} \alpha$ line ratio, dispersion, and velocity profiles taken in annuli containing 400 pixels centred on the AGN. Dispersions and velocities are given in $\mathrm{km} \mathrm{s}^{-1}$ and the distance from the AGN is in kpc.

gas flows down on either sides of the bubble, the highest velocities located just behind the bubble. Table 1 shows the comparisons between Hitomi and Sitelle best-fitted bulk velocities. Contrary to the velocity dispersions, we see no correlations between the bulk velocities of the warm and ionized gas, except for Reg 0 .

\section{CONCLUSION}

We have used SITELLE observations to probe the detailed dynamics of the filamentary nebula surrounding NGC 1275.

(i) We observe a smooth radial gradient of the $[\mathrm{N} \mathrm{III}] \lambda 6583 / \mathrm{H} \alpha$ line ratio, suggesting a change in the ionization mechanism and source across the nebula: higher line ratios are found in the central region and must therefore be related to energetic sources of ioniza-

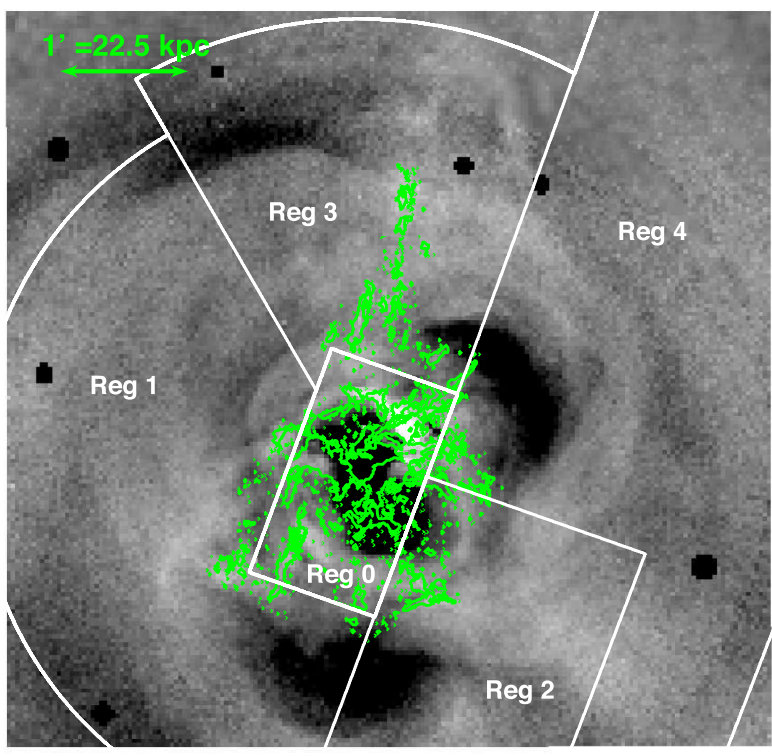

Figure 4. Chandra composite fractional residual image from Fabian et al. (2011) in the $0.5-7 \mathrm{keV}$ band (1.4 Ms exposure). The point spread function (PSF) corrected Hitomi regions from Hitomi Collaboration et al. (2017) are shown in white. Contours from the $\mathrm{H} \alpha$ flux map (starting at $3 \times 10^{-17}$ $\mathrm{erg} / \mathrm{s} / \mathrm{cm}^{2} / \mathrm{pixel}$ ) are shown in green.

Table 1. Comparison between Hitomi and Sitelle best-fitted bulk velocities and dispersions in regions shown in Fig.4.

\begin{tabular}{lcccc}
\hline & \multicolumn{2}{c}{ Hitomi } & \multicolumn{2}{c}{ SITELLE } \\
Region & $\begin{array}{c}v_{\text {bulk }} \\
\left(\mathrm{km} \mathrm{s}^{-1}\right)\end{array}$ & $\sigma_{v}\left(\mathrm{~km} \mathrm{~s}^{-1}\right)$ & $\begin{array}{c}v_{\text {bulk }} \\
\left(\mathrm{km} \mathrm{s}^{-1}\right)\end{array}$ & $\sigma_{v}\left(\mathrm{~km} \mathrm{~s}^{-1}\right)$ \\
\hline Reg 0 & $75_{-28}^{+26}$ & $189_{-18}^{+19}$ & $48 \pm 3$ & $145 \pm 3$ \\
Reg 1 & $46_{-19}^{+19}$ & $103_{-20}^{+19}$ & $-8 \pm 9$ & $155 \pm 9$ \\
Reg 2 & $47_{-14}^{+14}$ & $98_{-17}^{+17}$ & $182 \pm 2$ & $116 \pm 3$ \\
Reg 3 & $-39_{-16}^{+15}$ & $106_{-20}^{+20}$ & $122 \pm 2$ & $94 \pm 2$ \\
Reg 4 & $-77_{-28}^{+29}$ & $218_{-21}^{+21}$ & $182 \pm 3$ & $88 \pm 3$ \\
\hline
\end{tabular}




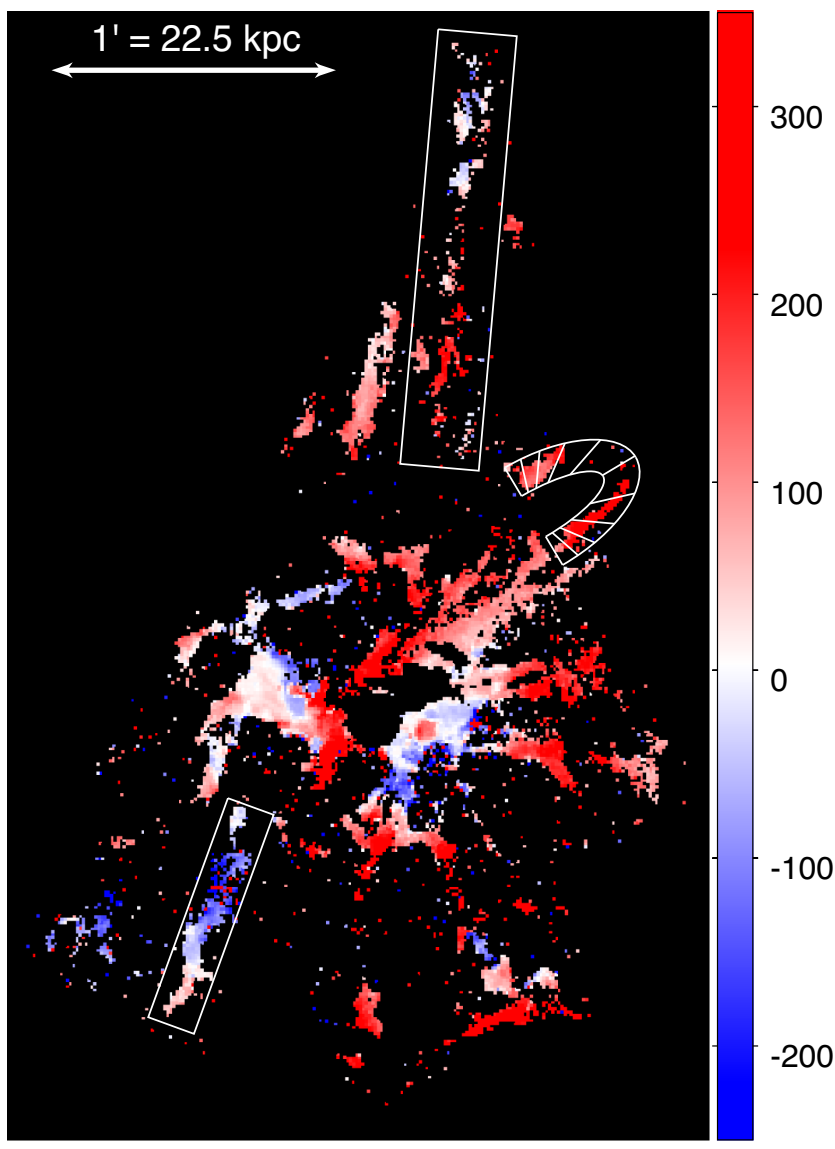

Figure 5. The velocity map of the nebula (in $\mathrm{km} \mathrm{s}^{-1}$ ). Profiles extracted from the three white regions are shown in Fig.6.
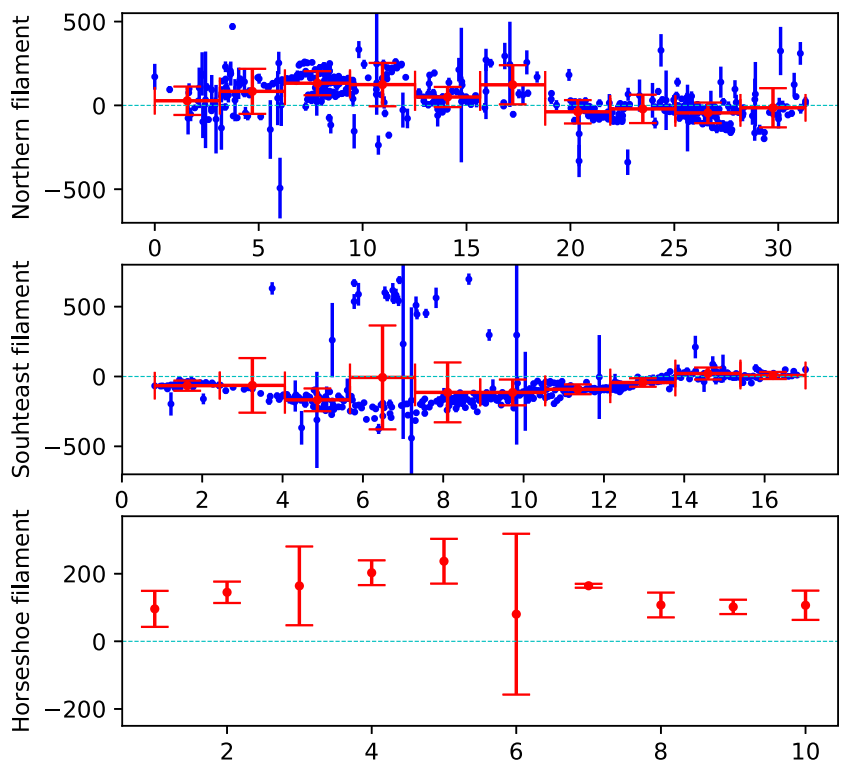

Figure 6. Velocity profiles extracted from the three white regions on Fig. 5 (in $\mathrm{km} \mathrm{s}^{-1}$ ). In the velocity profiles across the northern and the southeast filaments, velocities from each pixel are in blue while the means taken in 10 bins of equal width are shown in red. These are plot against their distance from the base of the filaments (in $\mathrm{kpc}$ ). A velocity profile across the horseshoe filament is also shown, the mean of the fitted velocities taken from 10 bins. tion (AGN and shocks), while filaments must be ionized by a source with lower power.

(ii) The velocity dispersions are as low and uniform as the Hitomi measurements of the ICM, while we see no bulk velocities correlations.

(iii) The velocity map of NGC 1275 revealed by SITELLE shows a previously unknown rich velocity structure across the entire nebula with no clear general trend or potential rotation, indicating that filaments are not falling uniformly onto the galaxy, nor being pulled out from it.

These results demonstrate how SITELLE, with its large field of view, high angular and spectral resolution, is well suited for the study of emission-line nebulae among clusters' BCGs.

\section{ACKNOWLEDGEMENTS}

MLGM is supported by the NSERC Postgraduate ScholarshipsDoctoral Program. JHL and LD are supported by NSERC through the discovery grant and Canada Research Chair programs, as well as FRQNT. ACF acknowledges ERC Advanced Grant 340442. ACE acknowledges support from STFC grant ST/P00541/1. This article is based on observations obtained at the Canada-France-Hawaii Telescope (CFHT) which is operated from the summit of Maunakea by the National Research Council of Canada, the Institut National des Sciences de l'Univers of the Centre National de la Recherche Scientifique of France, and the University of Hawaii. The observations at the Canada-France-Hawaii Telescope were performed with care and respect from the summit of Maunakea, which is a significant cultural and historic site.

\section{REFERENCES}

Baldwin J. A., Phillips M. M., Terlevich R., 1981, PASP, 93, 5

Canning R. E. A. et al., 2014, MNRAS, 444, 336

Conselice C. J., Gallagher III J. S., Wyse R. F. G., 2001, ApJ, 122, 2281

Crawford C. S., Allen S. W., Ebeling H., Edge A. C., Fabian A. C., 1999 , MNRAS, 306, 857

Fabian A. C., 2012, ARA\&A, 50, 455

Fabian A. C., Johnstone R. M., Sanders J. S., Conselice C. J., Crawford C. S., Iii J. S. G., Zweibel E., 2008, Nature, 454, 968

Fabian A. C., Sanders J. S., Crawford C. S., Conselice C. J., Gallagher J. S., Wyse R. F. G., 2003, MNRAS, 344, L48

Fabian A. C., Sanders J. S., Taylor G. B., Allen S. W., Crawford C. S., Johnstone R. M., Iwasawa K., 2006, MNRAS, 366, 417

Fabian A. C. et al., 2011, MNRAS, 418, 2154

Forman W., Kellogg E., Gursky H., Tananbaum H., Giacconi R., 1972, ApJ, 178,309

Gaspari M., Temi P., Brighenti F., 2017b, MNRAS, 466, 677

Gaspari M. et al., 2018, ApJ, 854, 2, 167

Gendron-Marsolais M. et al., 2017, MNRAS, 469, 3872

Hamer S. L. et al., 2016, MNRAS, 460, 1758

Hatch N. A., Crawford C. S., Fabian A. C., Johnstone R. M., 2006, MNRAS, 367,433

Hitomi Collaboration et al., 2016, Nature, 535, 117

Hitomi Collaboration et al., 2018, Publications of the Astronomical Society of Japan, 70, 2, 9

Ho I.-T., Lim J., Dinh-V-Trung, 2009, ApJ, 698, 1191

Kent S. M., Sargent W. L. W., 1979, ApJ, 230, 667

Kewley L. J., Groves B., Kauffmann G., Heckman T., 2006, MNRAS, 372, 961

Levenberg K., 1944, Quart. Appl. Math., 2, 164

Lim J., Ao Y., Dinh-V-Trung, 2008, ApJ, 672, 252

Lim J., Ohyama Y., Chi-Hung Y., Dinh-V-Trung Shiang-Yu W., 2012, ApJ, 744,112 
Lynds R., 1970, ApJL, 159

Marquardt D. W., 1963, SIAM J Appl Math, 11, 431

Martin T., Drissen L., Joncas G., 2015, Astronomical Data Analysis Software and Systems XXIV, 495:327

Martin T. B., Drissen L., Melchior A.-L., 2018, MNRAS, 473, 4130

Martin T. B., Prunet S., Drissen L., 2016, MNRAS, 463, 4223

McDonald M., Veilleux S., Rupke D. S. N., 2012, ApJ, 746, 153

McDonald M., Veilleux S., Rupke D. S. N., Mushotzky R., 2010, ApJ, 721, 1262
Minkowski, R., 1957, Proceedings 4th IAU Symposium, 4, 107

Russell H. R. et al., 2016, MNRAS, 458, 3134

Salomé P., Combes F., Revaz Y., Downes D., Edge A. C., Fabian A. C., 2011, A\&A, 531, A85

Salomé P. et al., 2006, A\&A, 454, 437

This paper has been typeset from a $\mathrm{T}_{\mathrm{E}} \mathrm{X} / \mathrm{LAT} \mathrm{E}$ file prepared by the author. 